\title{
Carfilzomib en el tratamiento de mieloma múltiple: revisión sistemática de la literatura y experiencia colombiana basada en RIPS
}

\author{
Carfilzomib for the Treatment of Multiple Myeloma: Systematic
} Review of the Literature and Colombian Experience Based on RIPS Carfilzomib no tratamento do mieloma múltiplo: revisão da literatura e da experiência colombiana baseada em RIPS

\author{
Camilo Castañeda, MD.* \\ María Camila Pantoja, MD.** \\ Luzmila Montaño, Enf.*** \\ Diego Rosselli, MD., EdM., MSc.****
}

\begin{abstract}
Resumen
Introducción: El mieloma múltiple es un tumor maligno y progresivo de las células B asociado a lesiones osteolíticas, inmunodeficiencia y disfunción renal que ha sido tratado desde 2008 con bortezomib con el cual se logró un aumento del tiempo de sobrevida de los pacientes, sin embargo su uso se vio limitado por efectos adversos dependientes de la dosis, en especial la neuropatía periférica dolorosa. Recientemente ha entrado en uso carfilzomib (agente inhibidor de proteosoma de segunda generación) aprobado por la FDA en 2012 como agente único para tratamiento de enfermedad refractaria y recaídas de mieloma múltiple. Objetivo: Realizar una revisión de la literatura y determinar la experiencia colombiana con el medicamento carfilzomib teniendo en cuenta las estadísticas nacionales consignadas en los registros individuales de prestación de servicios de salud. Además se describe la situación de un caso clínico. Metodología: La revisión se hizo con base en literatura indexada en PubMed; como criterio de inclusión se tuvo en cuenta que los artículos a revisar fueran estudios clínicos de fase 2 y 3 de carfilzomib en manejo de mieloma múltiple.
\end{abstract}

\begin{abstract}
Respecto a la experiencia colombiana se identificaron 37 pacientes colombianos con mieloma múltiple diagnosticado entre 2003 y 2012 y a quienes se hubiera prescrito carfilzomib, teniendo en cuenta información proporcionada por Biotoscana. Resultados: En total se localizaron 8 estudios clínicos con asignación al azar que evidencian efectos adversos no hematológicos como fatiga (367 de los 678 pacientes, $54 \%$ ), y náusea (323 pacientes, 48\%). En el caso de los RIPS, de los 37 pacientes, 22 pacientes recibieron de 1 a 8 ciclos de carfilzomib (promedio 3.0, mediana 3). Se evaluó la respuesta clínica en 9 de ellos, encontrándose 4 pacientes $(22 \%)$ en respuesta parcial muy buena, 4 pacientes $(22 \%)$ en respuesta parcial, 1 paciente $(6 \%)$ con enfermedad estable. Conclusiones: Se encontró diferencia entre los efectos adversos que se reportan en Colombia y los que se han reportado globalmente, predominando en nuestros pacientes la fatiga; asimismo se concluye que la experiencia con nuevos medicamentos no ha sido suficientemente documentada. [Castañeda C, Pantoja MC, Montaño L, Rosselli D. Carfilzomib en el tratamiento de mieloma múltiple: revisión sistemática de la literatura y experiencia colombiana basada en RIPS. MedUNAB 2015; 17(2):99-106]
\end{abstract}

* Médico, Residente de Neurología, Departamento de Neurociencias, Hospital Universitario San Ignacio, Pontificia Universidad Javeriana, Bogotá, Colombia. ** Estudiante de medicina, Facultad de Medicina, Pontificia Universidad Javeriana, Bogotá, Colombia.

*** Enfermera, Laboratorios Biotoscana, Bogotá, Colombia

${ }^{* * * *}$ Profesor asociado, Departamento de Epidemiología Clínica y Bioestadística, Facultad de Medicina, Pontificia Universidad Javeriana, Bogotá, Colombia.

Correspondencia: Diego Rosselli, Departamento de Epidemiología Clínica y Bioestadística, Facultad de Medicina, Pontificia Universidad Javeriana. Carrera 7 No.40-62, Hospital Universitario San Ignacio, piso2.E-mail diego.rosselli@gmail.com

Artículo recibido: 08 de julio de 2014, Aceptado: 28 de noviembre de 2014 
Palabras clave: Mieloma múltiple; Polineuropatías; Carfilzomib; Inhibidores de proteosoma; Bortezomib.

\begin{abstract}
Introduction: Multiple myeloma is a B-cell malignant and progressive tumor, associated with osteolytic lesions, immunodeficiency and renal dysfunction, that has been treated since 2008 with Bortezomib, which achieved an increase in patients survival, however its use was limited due to dose dependent adverse effects, especially painful peripheral neuropathy, it has recently come into use Carfilzomib (a second generation proteasome inhibitor agent) approved by the FDA in 2012 as the only agent for treatment of refractory disease and relapsing of multiple myeloma. Objective: To conduct a literature review and determine the Colombian experience with the drug Carfilzomib, considering national statistics contained in the individual records of health service delivery, plus the status of a case is described. Methodology: for the review was considered literature indexed in PubMed, as an inclusion criterion was considered clinical studies Phase 2 and 3 that reviewed Carfilzomib in handling multiple myeloma. Regarding the Colombian experience, taking information provided by Biotoscana, 37 multiple myeloma Colombian patients diagnosed between 2003 and 2012 and who had been prescribed Carfilzomib were identified. Results: A total of eight randomized trials shown on hematologic adverse effects such as fatigue (367 of 678 patients, $54 \%$ ), and nausea (323 patients, $48 \%$ ). For the RIPS of 37 patients, 22 patients received 1 to 8 cycles Carfilzomib (3.0 average, median 3). Clinical response in 9 of them, 4 patients (22\%) very good partial response, 4 patients $(22 \%)$ partial response, 1 patient $(6 \%)$ with stable disease was assessed. Conclusions: We found difference between the side effects reported in Colombia from the ones who have been reported globally, fatigue prevails in our patients, also experience with new drugs has not been sufficiently documented. [Castañeda C, Pantoja MC, Montaño $L$, Rosselli D. Carfilzomib for the Treatment of Multiple Myeloma: Systematic Review of the Literature and Colombian Experience Based on RIPS. MedUNAB 2015; 17(2):99-106]
\end{abstract}

Keywords: Multiple myeloma; Polyneuropathies; Carfilzomib; Proteosome inhibitors; Bortezomib.

\section{Introducción}

En 2008 la Administración de Alimentos y Medicamentos de Estados Unidos (Food and Drug Administration FDA) aprobó el uso de bortezomib, para el tratamiento de mieloma múltiple. Este fue el primero de una nueva clase de medicamentos: los inhibidores de proteosoma. E1 proteosoma es parte del sistema ubicuitina-proteosoma encargado de degradar proteínas celulares de una manera muy regulada. La inhibición del proteosoma, que es reversible en el caso del bortezomib, trae consigo altos índices de apoptosis de células malignas (1) y por consiguiente un mejor control de la enfermedad. Con el bortezomib se logró un importante aumento en el tiempo de sobrevida de los pacientes (2). El mieloma múltiple se

\section{Resumo}

Introdução: O mieloma múltiplo é um tumor maligno e progressivo das células B associado com lesões osteolíticas, imunodeficiência e disfunção renal que tem sido tratados desde 2008 com bortezomib com o qual se conseguiu um aumento no tempo de sobrevida dos pacientes, entretanto a seu uso foi limitado pelos efeitos colaterais dose dependente, especialmente a neuropatia periférica dolorosa. Recentemente se introduziu o uso Carfilzomib (agente inibidor do proteosoma de segunda geração), aprovado pelo FDA em 2012 como única droga para o tratamento da doença refratária e recidiva do mieloma múltiplo. Objetivo: Realizar revisão da literatura e determinar a experiência colombiana com a droga Carfilzomib considerando as estatísticas nacionais contidas nos prontuários individuais de prestação de serviços de saúde, além de descrever um caso clínico. Metodologia: Para a revisão foi levantada a literatura indexada no PubMed, sendo o critério de inclusão os estudos clínicos de fase 2 e 3 de Carfilzomib no tratamento do mieloma múltiplo. Em relação a experiência colombiana, foram identificados 37 pacientes colombianos com mieloma múltiplo diagnosticado entre 2003 e 2012 e que foram submetidos a prescrição de Carfilzomib, levando em conta as informações fornecidas pela BIOTOSCANA. Resultados: Foram localizados 8 estudos clínicos randomizados que evidenciaram efeitos colaterais não hematológicos tais como fadiga (367 de 678 pacientes, $54 \%$ ) e náuseas (323 pacientes, $48 \%$ ). Para os RIPS, 22 dos 37 pacientes receberam de 1 a 8 ciclos de Carfilzomib (média=3,0, mediana=3). Foi avaliada a resposta clínica em 9 pacientes, sendo $4(22 \%)$ com resposta parcial muito boa, 4(22\%) resposta parcial e 1 (6\%) com doença estável. Conclusões: Encontramos diferença entre os efeitos colaterais relatados na Colômbia em relação aos relatados mundialmente, com predominância da fadiga em nossos pacientes e se conclue que a experiência com novas drogas não foi suficientemente documentada. [Castañeda $C$, Pantoja MC, Montaño L, Rosselli D. Carfilzomib no tratamento do mieloma múltiplo: revisão da literatura e da experiência colombiana baseada em RIPS. MedUNAB 2015; 17(2):99-106]

Palavras-chave: Mieloma múltiplo; Polineuropatia; Carfilzomib; Inibidores de proteassoma; Bortezomib.

escogió como primer objetivo por ser un tumor maligno y progresivo de las células B, caracterizado por una acumulación anormal de células monoclonales en la médula ósea, asociada a lesiones osteolíticas, inmunodeficiencia y disfunción renal (3). La mayoría de los pacientes con mieloma múltiple se presentan con dolor óseo o fracturas patológicas, falla renal, infecciones recurrentes, anemia o fatiga. La mayoría de los casos son diagnosticados en personas mayores de 65 años, mientras que solo $1 \%$ de los casos se diagnostican en personas menores de 35 años (4).

A pesar del adelanto que representó el bortezomib, su empleo se vio limitado por sus efectos adversos dependientes de dosis, entre los cuales resalta la toxicidad hematológica y en más del 30\% de los casos la neuropatía periférica dolorosa (5). El tratamiento es, de todas maneras, 
paliativo ya que tarde o temprano los pacientes presentan recaídas y progresión de la enfermedad (6).

Las opciones terapéuticas para los pacientes en estadio avanzado incluyendo los que recaen después de múltiples líneas de tratamiento y aquellos con enfermedad refractaria han sido limitadas y las guías del National Comprehensive Cancer Network (NCCN) de 2011 recomiendan trasplante de medula ósea (7).

Carfilzomib se desarrolló como parte de una segunda generación de inhibidores de proteosoma; fue aprobado por la FDA en 2012 como agente único en el tratamiento para recaídas de mieloma múltiple o para enfermedad refractaria en pacientes que hayan recibido al menos dos terapias previas (2), incluyendo bortezomib y un agente inmunomodulador, y en quienes se haya demostrado progresión de la enfermedad durante los 60 días siguientes a la finalización de la última terapia.

La inhibición del proteosoma ha resultado un mecanismo efectivo $(8,9)$ no solo en el tratamiento del mieloma múltiple sino en otras discrasias hematológicas como macroglobulinemia de Waldenström, linfomas no Hodgkin de bajo grado y amiloidosis primaria (10).

Desde el punto de vista químico, carfilzomib es un esqueleto de tetrapéptido con una mitad de epoxicetona, análogo de epoxomicina, que logra una inhibición irreversible y más específica sobre el proteosoma $(11,12)$, lo cual explica la capacidad de carfilzomib en superar la resistencia que presenta bortezomib en estudios in vitro e in vivo (2). La irreversibilidad solo puede recuperarse sintetizando nuevas unidades proteosómicas. En dosis altas carfilzomib tiene un efecto adicional inhibitorio de los sitios similares a tripsina, a caspasa y a quimotripsina. Esta coinhibición, junto con el bloqueo de las subunidades del proteosoma lleva a un retraso en el crecimiento global celular en estudios in vitro (13).

Dentro de los efectos adversos menores (grado 1 y 2 ) se encuentran fatiga, náusea, disnea, diarrea, cefalea $(14,15)$. Como efectos adversos de mayor severidad (grado 3 / 4) se han descrito anemia, trombocitopenia, linfopenia, fatiga, neumonía y dolor $(16,17)$.

El objetivo del presente trabajo fue revisar la literatura indexada en PubMed que describiera estudios clínicos fases 2 y 3 de carfilzomib en mieloma múltiple y determinar la experiencia colombiana con el medicamento según las estadísticas nacionales consignadas en los RIPS (registros individuales de prestación de servicios de salud), y se describe un caso clínico seleccionado.

\section{Metodología}

Revisión de la literatura. Se empleó la siguiente estrategia de búsqueda sin restricciones de tiempo ni otros filtros en el portal de Pubmed: se utilizaron los siguientes términos "Multiple Myeloma" [All Fields] AND ("carfilzomib" [Supplementary Concept] OR "carfilzomib" [All Fields]). Dentro de los criterios de inclusión se tuvieron en cuenta solamente estudios clínicos con asignación al azar; en caso de estudios duplicados o de seguimiento se escogió la versión más reciente.

Los RIPS: La Resolución №3374 de 2000 estableció el Sistema de Información de las Prestaciones de Salud, cuyo elemento principal fue el registro individual de prestación de servicios de salud (conocidos como RIPS). Su propósito, además de allegar y centralizar la información en salud, fue facilitar la relación entre pagadores y prestadores proporcionando soportes de los servicios efectivamente prestados a la población en todos los regímenes definidos por la Ley 100. La información de los RIPS, cuya calidad ha ido mejorando en años recientes, constituye un subsistema de información prioritario para la evaluación y monitoreo del funcionamiento del Sistema General de Seguridad Social en Salud. Algunos trabajos, publicados en revistas indexadas $(18,19)$ han empleado los RIPS con diferentes fines. Para este estudio se revisaron los RIPS con el diagnóstico de "mieloma múltiple" (Código CIE-10 C900, dentro de la categoría C81-C96 “tumores malignos del tejido linfático, de los órganos hematopoyéticos y de tejidos afines"). Las estadísticas revisadas corresponden a los años 2009-2012, divididos por grupos etarios y por sexo. A esta información se puede acceder a través del vínculo "cubos.sispro.gov.co", empleando una clave de acceso suministrada con los respectivos permisos por la Dirección General de Planeación y Análisis de Política del Ministerio de Salud.

Experiencia colombiana: Se revisaron las historias clínicas de todos los pacientes colombianos en quienes se ha prescrito carfilzomib y se ha hecho la solicitud de su aprobación al Invima para hacer una caracterización y descripción de esta muestra limitada de pacientes. A esta base de datos se tuvo acceso a partir de información recogida por Biotoscana con los respectivos permisos.

\section{Resultados}

Revisión de la literatura: La Figura 1 muestra el proceso de selección de los estudios clínicos publicados en revistas indexadas. En total se encontraron 8 estudios clínicos aleatorizados $(15,17,5)$, publicados entre los años 2009 y 2014 que reunen un total de 678 pacientes con una edad media de 63.6 años (rango 21 a 87) y con ligero predominio masculino (57.5\%) y en su mayoría caucásicos (ver Tabla 1$)$. Dos de los estudios $(22,23)$ incluyeron pacientes con otras enfermedades como macroglobulinemia de Waldeström, linfoma no Hodgkin y linfoma Hodgkin. De los 8 estudios, 6 clasificaron a los pacientes según la escala de rendimiento Eastern Cooperative Oncology Group (ECOG) $(15,17,20,21,24,25)$, en ellos la mayoría de los pacientes se encontraban en ECOG 1 (restringidos para actividad 


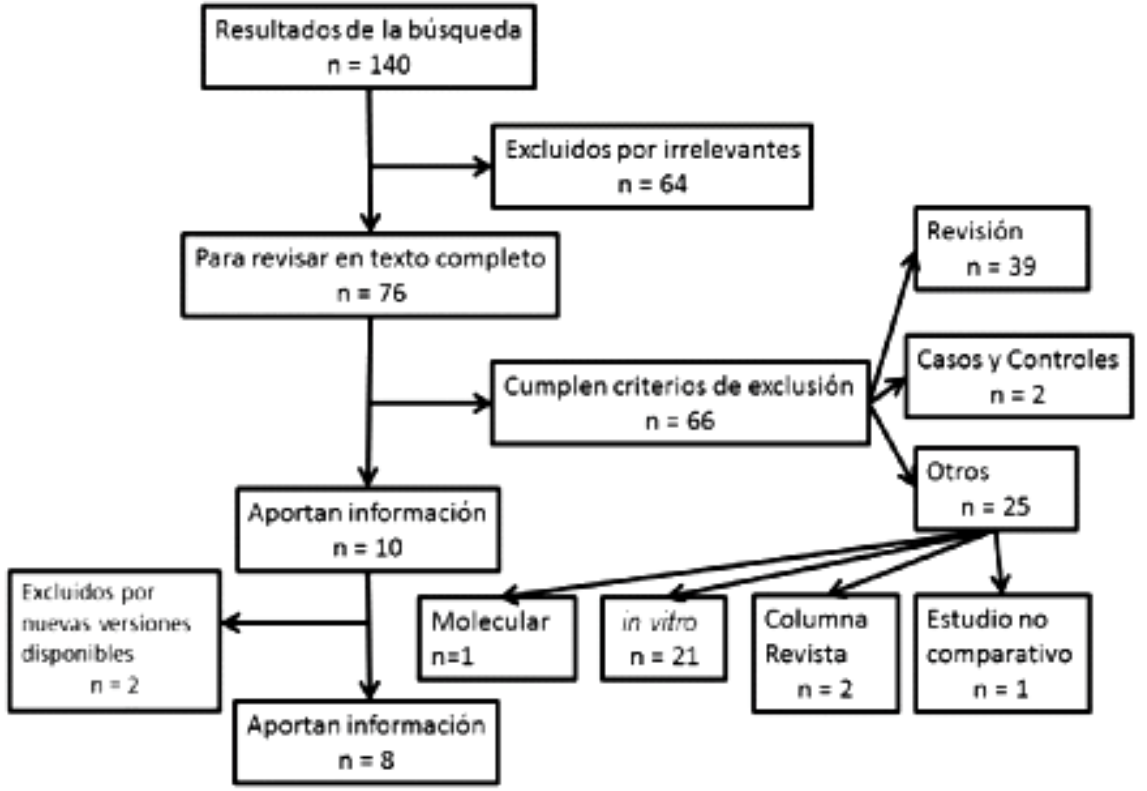

Figura 1. Proceso de selección de los artículos

Tabla 1. Características generales de los pacientes incluidos en los 8 estudios clínicos

\begin{tabular}{|c|c|c|c|c|c|c|c|c|}
\hline Autor & Jagannath & Niesvizky & Berenson & Alisina* & Jakuboniak & Siegel & Vij & Owen * \\
\hline Año & 2012 & 2013 & 2014 & 2012 & 2013 & 2012 & 2012 & 2009 \\
\hline$N$ & 46 & 40 & 38 & 28 & 229 & 266 & 35 & 10 \\
\hline Hombres & $25(54 \%)$ & $22(55 \%)$ & $25(66 \%)$ & $(54.1 \%)^{*}$ & $132(58 \%)$ & $155(58 \%)$ & $18(51 \%)$ & $(69 \%)$ \\
\hline \multicolumn{9}{|l|}{ Edad } \\
\hline media & 63.5 & 61.5 & 67 & 62.3 & 64.4 & 63 & 63 & 62.4 \\
\hline rango & $44-82$ & $43-81$ & $47-79$ & $25-82$ & $37-87$ & $37-87$ & $40-77$ & $21-85$ \\
\hline \multicolumn{9}{|l|}{ Etnia } \\
\hline caucásicos & $34(74 \%)$ & $30(75 \%)$ & $29(76.3 \%)$ & 87.4 & & $190(71 \%)$ & & $23(79 \%)$ \\
\hline negros & $8(17 \%)$ & $6(15 \%)$ & $2(5.3 \%)$ & 6.6 & & $53(20 \%)$ & & $4(14 \%)$ \\
\hline hispanos & $1(2 \%)$ & $3(8 \%)$ & $1(2.6 \%)$ & 2.0 & & $10(4 \%)$ & & $2(7 \%)$ \\
\hline otros & $3(7 \%)$ & $1(3 \%)$ & $1(2.6 \%)$ & 4.0 & & $13(5 \%)$ & & 0 \\
\hline \multicolumn{9}{|l|}{ ECOG } \\
\hline 0 & $12(26 \%)$ & $10(25 \%)$ & $17(45 \%)$ & & $62(27 \%)$ & $69(26 \%)$ & $15(43 \%)$ & \\
\hline 1 & $26(57 \%)$ & $29(72 \%)$ & $17(45 \%)$ & & $139(61 \%)$ & $162(61 \%)$ & $19(54 \%)$ & \\
\hline 2 & $7(15 \%)$ & $1(3 \%)$ & $4(10 \%)$ & & $28(12 \%)$ & $35(13 \%)$ & & \\
\hline Neuropatía \% & $87 \%$ & & $16 \%$ & $14 \%$ & $12 \%$ & $77 \%$ & $54 \%$ & $30 \%$ \\
\hline \multicolumn{9}{|c|}{ Medicamentos previos \% } \\
\hline bortezomib & $46(100 \%)$ & $30(75 \%)$ & $38(100 \%)$ & & $228(99.5 \%)$ & $265(100 \%)$ & $35(100 \%)$ & \\
\hline lenalidomida & $42(91 \%)$ & $28(70 \%)$ & $6(16 \%)$ & & $229(100 \%)$ & $249(94 \%)$ & $13(37 \%)$ & \\
\hline talidomida & $42(91 \%)$ & $20(50 \%)$ & $2(5 \%)$ & & & $199(75 \%)$ & $24(69 \%)$ & \\
\hline
\end{tabular}

* Estos estudios incluyeron pacientes con otras neoplasias, se presentan solo los de mieloma múltiple 
extenuante); 7 de los 8 estudios $(20,21,23-27)$ evaluaron la presencia de neuropatía al ingreso al estudio según la escala Common Terminology Criteria for Adverse Events (CTCAE) $(15,17,20,22-25)$. En general se encontró que la mayoría de los pacientes no tenían neuropatía (grado 0), excepto en el estudio de Jagannath (17). Cuando había neuropatía era grado 1 (asintomática, diagnosticada por observaciones clínicas o diagnósticas) o grado 2 (sintomatología moderada, limita las actividades instrumentales de la vida cotidiana). En 6 de los estudios $(15,17,20,21,24,25)$ se mencionan los medicamentos previamente usados, el más común fue bortezomib (en 642 de 654 pacientes, $98 \%$ ), seguido por lenalidomida (en 567 de $654,87 \%$ ) y talidomida ( 287 de $425,68 \%$ ).

De los 678 pacientes, se evaluó la respuesta al tratamiento tumoral de mieloma múltiple con carfilzomib en 633 pacientes (93\%). Se clasificaron en 6 categorías de respuesta desde respuesta completa estricta hasta enfermedad progresiva. En los 8 artículos se encontraron 7 pacientes con respuesta completa estricta (1\%); 46 pacientes $(7 \%)$ presentaron respuesta "muy buena parcial"; 124 pacientes (20\%) presentaron respuesta parcial; 87 pacientes $(14 \%)$ respuesta mínima; 197 pacientes (31\%) permanecieron estables; 172 pacientes $(27 \%)$ presentaron progresión de la enfermedad. La respuesta global (overall response rate), definida como respuesta parcial o mejor se presentó en 177 de los 633 pacientes (28\%), y osciló entre el $17-63 \%$ en todos los estudios. La duración de esta respuesta fue entre 7 y 12 meses. El beneficio clínico (clinical benefit response), definido como respuesta mínima o mejor, se encontró en 264 de los 605 pacientes en que se evaluó (44\%), y varió entre 31 y $75 \%$.
Como principales efectos adversos hematológicos se recalcan en los 8 estudios anemia (descrita en 328 de los 678 pacientes, 48\%), trombocitopenia (en 275 pacientes, $41 \%$ ), neutropenia solo fue reportada en 7 estudios (y se encontró en 92 de 449,20\%) y linfopenia reportada en 5 estudios (111 de 411 pacientes, 27\%). Dentro de los efectos adversos no hematológicos se encuentran en los 8 estudios fatiga ( $367 \mathrm{de}$ los 678 pacientes, $54 \%$ ), y náusea (323 pacientes, $48 \%$ ). Otros eventos adversos descritos fueron diarrea en 7 estudios (entre $27 \%$ y $53 \%$ ), y 2 estudios reportaron tos (28$43 \%$ ) o disnea (34-37\%). En un estudio (17) se describió infección de vías respiratorias altas en el $40 \%$ de los pacientes (17 de 42), y en otro (24) hiperglicemia en 49\% de los pacientes (18 de 37 pacientes).

Los RIPS: La Tabla 2 muestra los datos oficiales correspondientes al diagnóstico de mieloma múltiple en Colombia, según grupos etarios (quinquenios DANE) y según sexo. Si se excluyen los casos en menores de 40 años que son improbables (y que equivalen a $8.5 \%$ del total de registros) y se calcula la prevalencia neta se obtiene un valor de 4.5 en mujeres y 4.2 en hombres por cada 100,000 habitantes.

La experiencia colombiana: Se estudiaron en total 37 pacientes colombianos (19 hombres, 18 mujeres) con mieloma múltiple diagnosticado entre 2003 y 2012 y a quienes se hubiera prescrito carfilzomib (ver Tabla 3). Los pacientes estaban siendo tratados en 11 ciudades (Armenia, Barranquilla, Bogotá, Cali, Cúcuta, Medellín, Montería, Pereira, Popayán, Santa Marta y Sincelejo). Los pacientes estaban afiliados a 13 Entidades Promotoras de Salud (EPS), uno pertenecía a las fuerzas militares y dos eran particulares.

Tabla 2. Casos de mieloma múltiple en Colombia según los reportes oficiales de los RIPS 2009-2012 para hombres $(\mathrm{H})$ y mujeres $(\mathrm{M})$

\begin{tabular}{|c|c|c|c|c|c|c|c|c|c|c|c|c|}
\hline \multirow[b]{2}{*}{ Edad (años) } & \multicolumn{3}{|c|}{2009} & \multicolumn{3}{|c|}{2010} & \multicolumn{3}{|c|}{2011} & \multicolumn{3}{|c|}{2012} \\
\hline & $M$ & $\mathrm{H}$ & Total & $M$ & $\mathrm{H}$ & Total & $M$ & $\mathrm{H}$ & Total & $M$ & $\mathrm{H}$ & Total \\
\hline De 0 a 39 & 70 & 72 & 142 & 83 & 71 & 154 & 61 & 72 & 133 & 76 & 76 & 152 \\
\hline De 40 a 44 & 30 & 23 & 53 & 34 & 30 & 64 & 38 & 25 & 63 & 37 & 31 & 68 \\
\hline De 45 a 49 & 33 & 40 & 73 & 42 & 46 & 88 & 52 & 56 & 108 & 70 & 48 & 118 \\
\hline De 50 a 54 & 73 & 72 & 145 & 70 & 93 & 163 & 75 & 102 & 178 & 103 & 114 & 217 \\
\hline De 55 a 59 & 75 & 75 & 151 & 105 & 90 & 195 & 123 & 115 & 238 & 138 & 134 & 272 \\
\hline De 60 a 64 & 71 & 73 & 144 & 114 & 130 & 244 & 117 & 127 & 244 & 162 & 160 & 322 \\
\hline De 65 a 69 & 78 & 78 & 156 & 110 & 95 & 205 & 115 & 118 & 234 & 161 & 153 & 315 \\
\hline De 70 a 74 & 77 & 56 & 133 & 109 & 100 & 209 & 129 & 117 & 246 & 156 & 148 & 305 \\
\hline De 75 a 79 & 61 & 56 & 117 & 93 & 80 & 173 & 115 & 74 & 189 & 131 & 79 & 211 \\
\hline De 80 ó más & 56 & 36 & 92 & 72 & 64 & 136 & 100 & 74 & 175 & 106 & 102 & 208 \\
\hline \multirow[t]{2}{*}{ Total $>40$ años } & 55 & 50 & 1064 & 749 & 728 & 1477 & 864 & 808 & 1675 & 106 & 969 & 2036 \\
\hline & 4 & 9 & & & & & & & & 4 & & \\
\hline \multirow[t]{2}{*}{ Total general } & 62 & 58 & 1206 & 832 & 799 & 1631 & 925 & 880 & 1808 & 114 & 104 & 2188 \\
\hline & 4 & 1 & & & & & & & & 0 & 5 & \\
\hline
\end{tabular}


Tabla 3. Características generales de la muestra de pacientes colombianos con diagnóstico de mieloma múltiple a quienes se les ha prescrito carfilzomib

\begin{tabular}{lc}
\hline Edad (años) & \\
\hline Rango & $48-65$ años \\
Promedio & 67 años \\
Mediana & 68 años \\
\hline Tratamientos previos & \\
\hline Dexametasona & $37(100 \%)$ \\
\hline Bortezomib & $36(97 \%)$ \\
\hline Agente alquilante & $33(89 \%)$ \\
\hline Lenalidomida & $30(81 \%)$ \\
\hline Talidomida & $29(28 \%)$ \\
\hline Doxorrubicina liposomal & $11(30 \%)$ \\
VAD* & $9(24 \%)$ \\
Trasplante de médula ósea & $6(16 \%)$ \\
\hline **ECOG al prescribir carfilzomib & \\
\hline I & $9(24 \%)$ \\
II & $9(24 \%)$ \\
III & $14(38 \%)$ \\
IV & $5(14 \%)$ \\
\hline
\end{tabular}

* VAD: vincristina, adriamicina, dexametasona

** ECOG: Eastern Cooperative Oncology Group

Fuente: Datos de la investigación

De los 37 pacientes, 22 recibieron de 1 a 8 ciclos de carfilzomib (promedio 3.0, mediana 3). Se evaluó la respuesta clínica en 9 de ellos, encontrándose 4 pacientes $(22 \%)$ en respuesta parcial muy buena, 4 pacientes $(22 \%)$ en respuesta parcial, 1 paciente $(6 \%)$ con enfermedad estable. De los 26 pacientes que continúan vivos, se tiene el dato de la fecha del diagnóstico de mieloma múltiple en 24 de ellos; el tiempo de seguimiento promedio ha sido de cuatro años y medio (rango 2 a 9 años; mediana 4 años).

La edad de diagnóstico de la enfermedad, en los pacientes de la muestra, estuvo comprendida entre 43 y 81 años (media 61.5; desviación estándar de 10.3; mediana 60). De los pacientes, 11 ya habían fallecido ( 1 de ellos antes de recibir el primer ciclo de carfilzomib) y 6 pacientes habían suspendido el tratamiento por diferentes razones ( 2 de ellos por progresión del mieloma, 1 de ellos por infarto agudo de miocardio y posible progresión del mieloma, 1 por enfermedad estable, 1 por infarto cerebral, y 1 por voluntad del paciente).

Sobre los efectos adversos reportados, el más frecuente fue fatiga, en 21 pacientes (91\%); otros fueron disnea en 12 pacientes $(52 \%)$, artralgias y mialgias en $11(48 \%)$, cefalea en $9(39 \%)$, edema facial en $4(17 \%)$, náusea o vómito en 4
$(17 \%)$, anemia en $4(17 \%)$, diarrea en $3(13 \%)$, y trombocitopenia en 1 paciente. En otro paciente se reportó vértigo $\mathrm{y}$ prurito los cuales no fueron considerados asociados a carfilzomib por el médico tratante.

\section{Conclusiones}

Según la revisión de la literatura que se realizó, de los 633 pacientes incluidos en los 8 estudios en quienes se evaluó la respuesta, tan solo algo más de una cuarta parte presentaron una respuesta global (overall response rate) equivalente a una respuesta parcial o mejor. Para la población colombiana solo se evaluó la respuesta clínica en nueve de 37 pacientes, así que el hecho de que 8 hayan presentado una respuesta parcial debe considerarse con cautela. Tanto en la experiencia colombiana como en la literatura, los pacientes llegan a recibir carfilzomib después de haber fallado a otras terapias como bortezomib, talidomida y lenalidomida.

En cuanto a la experiencia colombiana se encontró que los efectos adversos de nuestros pacientes difieren de los descritos en los estudios clínicos ya que en nuestro medio predominó la fatiga, mientras que los estudios internacionales enfatizan en los hallazgos de laboratorio como anemia, trombocitopenia y linfopenia.

De la experiencia colombiana también se puede concluir que la validez de los RIPS como fuente de información epidemiológica puede ser cuestionada. Eso se observa en el número de pacientes jóvenes, incluso niños con este diagnóstico seguramente equivocado. También llama la atención el incremento progresivo del diagnóstico en los 4 años del análisis, lo cual se podría interpretar como reducción del subregistro más que un aumento de incidencia. Sin embargo, a pesar de esta limitación, nuestros datos de prevalencia (4.5 por 100,000 en mujeres y 4.2 en hombres) podrían ser acertados ya que no distan mucho de lo reportado en la literatura. En Europa la prevalencia de la enfermedad se ha estimado en 6 por 100,000 (26); en el Japón 3.5 por 100,000 (27).

Este trabajo tiene como limitación el empleo de diferentes metodologías combinadas en un solo escrito: una presentación de un caso clínico seleccionado, una revisión de la literatura y un análisis de dos fuentes de información colombianas, ambas limitadas.

En conclusión, la experiencia colombiana en mieloma múltiple y particularmente con nuevos medicamentos no ha sido suficientemente documentada. Este trabajo contribuye al conocimiento en este campo.

\section{Anexo}

Una mujer de 67 años, natural de Génova Quindío y residente en Barranquilla, consultó en agosto de 2008 por disestesia en región lumbar de instauración aguda 
asociada a debilidad en miembros inferiores, por lo cual presentó caída desde su propia altura. Este dolor se mantuvo durante 20 días y empeoraba con el movimiento, dificultaba la marcha y no cedía con analgésicos. La resonancia magnética realizada un mes después mostró fractura por acuñamiento de L1 así como múltiples lesiones líticas en cuerpos vertebrales de columna torácica. Tenía además anemia normocítica normocrómica.

En junio de 2010 se le practicó inmunoelectroforesis en orina y se encontró gammapatía monoclonal de predominio de cadenas kappa. También se le practicó un mielograma con el cual se hizo diagnóstico de mieloma múltiple. Se inició manejo con talidomida $100 \mathrm{mg}$ día, analgésicos, ácido acetilsalicílico, doxorrubicina liposomal, dexametasona, ácido zoledrónico y eritropoyetina manteniéndose durante 2 años, con control por hemograma que mostró mejoría de anemia, con línea blanca celular normal y recuento de plaquetas normales. La bioquímica sanguínea mostró proteínas dentro de límite normal, inmunoglobulinas con un predominio de lgG con una IgM y una IgAen límites normales.

En septiembre de 2012 la paciente volvió a consultar por dolor y dificultad para la marcha. Los paraclínicos mostraban actividad de la enfermedad con proteína de Bence Jones positiva en orina, con predominio de cadenas kappa. El hemograma de control mostró anemia normocítica normocrómica, plaquetas y leucocitos dentro de límites normales. Se realizó una nueva radiografía de columna que documentó osteopenia con fractura por acuñamiento de todos los cuerpos lumbares más acentuado en L1. Sobre las proteínas en sangre, se encontró albúmina normal con alfa 1 globulina normal, alfa 2 globulina normal, beta globulinas normal y gammaglobulinas normales. Se realizó un mielograma que no mostró hallazgos de malignidad.

Por persistencia de actividad se cambió el medicamento a lenalidomida, sin embargo desarrolló alergia tipo III por lo cual se cambió a bortezomib con ácido ibandrónico. En febrero de 2013 presentó una nueva caída de su propia altura con fractura trocantérica izquierda que requirió reducción abierta con material de osteosíntesis. En esa ocasión los paraclínicos mostraban progresión de la enfermedad, con proteína de Bence Jones positiva, con gammapatía monoglonal kappa, persistencia de anemia normocítica normocrómica con recuento de plaquetas y línea blanca normales. Se decidió continuar con doxorrubicina liposomal con lenalidomida a dosis más bajas mientras se le aprobaba carfilzomib. En la actualidad (mayo 2014) lleva 6 ciclos de carfilzomib, refiere posterior a la aplicación del medicamento mareo, malestar generalizado y diaforesis de 1 día de duración. En marzo de 2014 se encontró inmunofijación de proteínas negativa en orina por primera vez. Sin embargo en el segundo control presentó nueva positivización, con predominio de cadenas kappa en orina cuantificadas en $668 \mathrm{mg} / \mathrm{ml}$. El último hemograma no muestra anemia, leucocitos en límite superior de la normalidad con recuento plaquetario normal. Actualmente la paciente puede caminar sin ayuda, realizar actividades domésticas como cocinar y barrer y desplazarse por el vecindario.

\section{Conflicto de intereses}

Este trabajo recibió el apoyo económico de Biotoscana.

\section{Referencias}

1. Siegel DS, Martin T, Wang M, Vij R, Jakubowiak AJ, Lonial S, et al. A phase 2 study of single-agent carfilzomib ( PX-171-003-A1) in patients with relapsed and refractory multiple myeloma. Blood. 2014 Oct 4;120(14):2817-25.

2. Redic K. Carfilzomib: a novel agent for multiple myeloma. J Pharm Pharmacol. 2013 Aug;65(8):1095-106.

3. Raab MS, Podar K, Breitkreutz I, Richardson PG, Anderson KC. Multiple myeloma. Lancet. 2009 Jul 25;374(9686):324-39.

4. Kyle R, Gertz M, Witzig TE, Lust J, Lacy MQ, Dispenzieri A, et al. Review of 1027 patients with newly diagnosed multiple myeloma. Mayo Clin Proc. 2003 Jan;78(1):2133.

5. Richardson PG, Barlogie B, Berenson J, Singhal S, Jagannath S, Irwin D, et al. A phase 2 study of bortezomib in relapsed, refractory myeloma. N Engl J Med. 2003 June 26;348:2609-17.

6. Kumar SK, Lee JH, Lahuerta JJ, Morgan G, Richardson PG, Crowley J, et al. Risk of progression and survival in multiple myeloma relapsing after therapy with IMiDs and bortezomib: a multicenter international myeloma working group study. Leukemia. 2012 Jan;26(1):149-57.

7. Anderson KC, Alsina M, Bensinger $\mathrm{W}$, Biermann JS, Chanan-Khan A, Cohen AD, et al. NCCN clinical practice guidelines in oncology: multiple myeloma. J Natl Compr Canc Netw. 2009 Oct 7;7(9):908-42.

8. Demo SD, Kirk CJ, Aujay M, Buchholz TJ, Dajee M, Ho $\mathrm{MN}$, et al. Antitumor activity of PR-171, a novel irreversible inhibitor of the proteasome. Cancer Res. 2007 Jul 1;67(13):6383-91.

9. Kuhn DJ, Orlowski RZ. The immunoproteasome as a target in hematologic malignancies. Semin Hematol. 2012 Jul;49(3):258-62.

10. Rajkumar SV, Richardson PG, Hideshima T, Anderson $\mathrm{KC}$. Proteasome inhibition as a novel therapeutic target in human cancer. J Clin Oncol. 2005 Jan 20;23(3):630-9.

11. Kim HB, Myung J, Sin N, Crews CM. Proteasome inhibition by the natural products epoxomicin and dihydroeponemycin: insights into specificity and potency. Bioorg Med Chem Lett. 1999 Dec 6;9(23):3335-40.

12. Meng L, Kwok BH, Sin N, Crews CM. Eponemycin exerts its antitumor effect through the inhibition of proteasome function. Cancer Res. 1999 Jun 15;59(12):2798-801. 
13. Arastu-Kapur S, Anderl JL, Kraus M, Parlati F, Shenk KD, Lee SJ, et al. Nonproteasomal targets of the proteasome inhibitors bortezomib and carfilzomib: a link to clinical adverse events. Clin Cancer Res. 2011 May 1;17(9):2734-43.

14. Singhal S, Siegel DS, Martin T, Vij R, Wang L, Jakubowiak AJ, et al. Integrated safety from phase 2 studies of monotherapy carfilzomib in patients with relapsed and refractory multiple myeloma (MM): an updated analysis. Poster session presented at: Myeloma - Therapy, excluding Transplantation. Proceedings of the 53rd ASH Annual Meeting \& Exposition; 2011 Dec 10; San Diego, CA.

15. Siegel D, Martin T, Nooka A, Harvey RD, Vij R, Niesvizky $\mathrm{R}$, et al. Integrated safety profile of single-agent carfilzomib: experience from 526 patients enrolled in 4 phase II clinical studies. Haematologica. 2013 Nov;98(11):1753-61.

16. Nooka AK, Badros AZ, Patel P, McCulloch L, Lonial S, Kaufman JL.. Hematologic safety data from four phase II studies of single-agent carfilzomib in relapsed and/or refractory multiple myeloma. Poster session presented at: General Poster Session, Lymphoma and Plasma Cell Disorders. Proceedings of the 2012 ASCO Annual Meeting. J Clin Oncol. 2012;30 Suppl; abstr 8086.

17. Jagannath S, Vij R, Stewart AK, Trudel S, Jakubowiak AJ, Reiman T, et al. An open-label single-arm pilot phase II study (PX- 171-003-A0) of low-dose, single-agent carfilzomib in patients with relapsed and refractory multiple myeloma. Clin Lymphoma Myeloma Leuk. 2012 Oct;12(5):310-8.

18. Rosselli D, Rueda JD. Burden of pneumococcal infection in adults in Colombia. J Infect Public Health. 2012 Oct;5(5):354-9.

19. Bernal O, Forero JC, Villamil P, Pino R. Disponibilidad de datos y perfil de morbilidad en Colombia. Rev Panam Salud Pública. 2012;31(3):181-7. Español.
20. Jakubowiak a J, Siegel DS, Martin T, Wang M, Vij R, Lonial S, et al. Treatment outcomes in patients with relapsed and refractory multiple myeloma and high-risk cytogenetics receiving single-agent carfilzomib in the PX171-003-A1 study. Leukemia. 2013 Dec;27(12):2351-6.

21. Niesvizky R, Martin TG, Bensinger WI, Alsina M, Siegel DS, Kunkel L, et al. Phase lb dose-escalation study (PX171-006) of carfilzomib, lenalidomide, and low-dose dexamethasone in relapsed or progressive multiple myeloma. Clin Cancer Res. 2013 Apr 15;19(8):2248-56.

22. O'Connor OA, Stewart AK, Vallone M, Molineaux CJ, Kunkel LA, Gerecitano JF, et al. A phase 1 dose escalation study of the safety and pharmacokinetics of the novel proteasome inhibitor carfilzomib (PR-171) in patients with hematologic malignancies. Clin Cancer Res. 2009 Nov 15;15(22):7085-91.

23. Alsina M, Trudel S, Furman RR, Rosen PJ, O'Connor O a, Comenzo RL, et al. A phase I single-agent study of twice-weekly consecutive-day dosing of the proteasome inhibitor carfilzomib in patients with relapsed or refractory multiple myeloma or lymphoma. Clin Cancer Res. 2012 Sep 1;18(17):4830-40.

24. Berenson JR, Hilger JD, Yellin O, Dichmann R, PatelDonnelly D, Boccia RV, et al. Replacement of bortezomib with carfilzomib for multiple myeloma patients progressing from bortezomib combination therapy. Leukemia. 2014 Jul;28(7):1529-36.

25. Vij R, Siegel DS, Jagannath S, Jakubowiak AJ, Stewart AK, McDonagh K, et al. An open-label, single-arm, phase 2 study of single-agent carfilzomib in patients with relapsed and/or refractory multiple myeloma who have been previously treated with bortezomib. $\mathrm{Br} \mathrm{J}$ Haematol. 2012 Sep 1;158(6):739-48.

26. Becker N. Epidemiology of multiple myeloma. Recent Results Cancer Res. 2011;183:25-35.

27. Suzuki K. Current therapeutic strategy for multiple myeloma. Jpn J Clin Oncol. 2013;43(2):116-24 